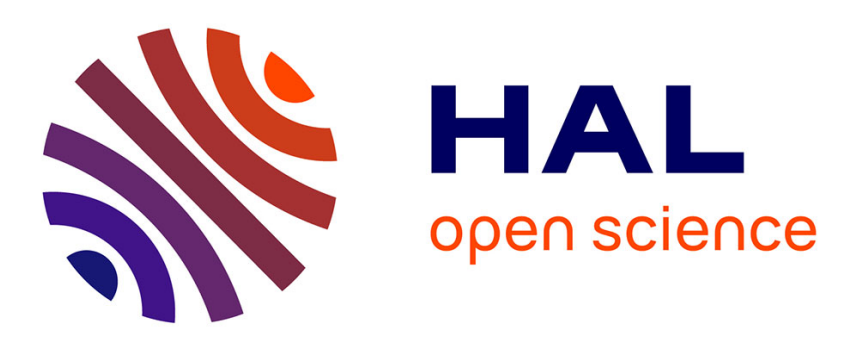

\title{
Determination of Timed Transitions in Identified Discrete-Event Models for Fault Detection
}

\author{
Stefan Schneider, Lothar Litz, Jean-Jacques Lesage
}

\section{To cite this version:}

Stefan Schneider, Lothar Litz, Jean-Jacques Lesage. Determination of Timed Transitions in Identified Discrete-Event Models for Fault Detection. 51st IEEE Annual Conference on Decision and Control (CDC'12), Dec 2012, Grand Wailea, Maui, HI (USA), United States. pp. 5816-5821. hal-00823582

\section{HAL Id: hal-00823582 https://hal.science/hal-00823582}

Submitted on 17 May 2013

HAL is a multi-disciplinary open access archive for the deposit and dissemination of scientific research documents, whether they are published or not. The documents may come from teaching and research institutions in France or abroad, or from public or private research centers.
L'archive ouverte pluridisciplinaire HAL, est destinée au dépôt et à la diffusion de documents scientifiques de niveau recherche, publiés ou non, émanant des établissements d'enseignement et de recherche français ou étrangers, des laboratoires publics ou privés. 


\title{
Determination of Timed Transitions in Identified Discrete-Event Models for Fault Detection
}

\author{
Stefan Schneider ${ }^{1}$, Lothar Litz ${ }^{1}$ and Jean-Jacques Lesage ${ }^{2}$
}

\begin{abstract}
Model-based fault detection compares modeled and observed behavior to decide whether a system operates properly or not. The key issue in this paper is to model large-scale Discrete Event Systems (DESs) with little a-priori knowledge. For this class of systems a new approach to blackbox determination of timed transitions for timed automata is proposed. The method identifies a set of time guards leading to an advantageous trade-off between the fault detection errors: false alarms and missed detections. A real industrial system is applied for evaluation of time guard determination. It is shown that applying the proposed method results in a better trade-off between the fault detection errors than using common methods (e.g. Min/Max, Normal Distribution).
\end{abstract}

\section{INTRODUCTION}

Productivity and availability are highly significant for industrial systems. Model-based fault diagnosis is an important technology to comply with these challenges. The concerned aspect in this work is the determination of a timed fault-free system model for fault detection purpose.

Several approaches of model-based fault diagnosis and fault detection in particular for Discrete Event Systems (DESs) evolved in the past. Only a few of them incorporate time information. In [1] condition templates are used to represent the system behavior with regard to event sequences and observation times. Timed fault detection is performed by observing whether an event occurred in an expected time interval or not. A method to determine these intervals based on confidence spaces is given in [2]. Time delays are considered as normally distributed. A combination of the diagnoser approach according to [3] with time information is presented in [4]. Tick events are used to describe the time between successive events. The diagnoser estimates the system state after each new observation and monitors the time to detect deadlocks.

The system models of these diagnosis approaches are knowledge based and therefore comprehensive a-priori information is required. For large-scale DESs this knowledge is particularly difficult to access and practically not available. One way to compete with this challenge is model identification. An identification method for DESs is described in [5]. The untimed fault-free behavior of the DES is reproduced by an automaton. In [6] the fault diagnosis capabilities using an identified model from [5] are compared with the diagnoser

\footnotetext{
${ }^{1}$ Stefan Schneider and Lothar Litz are with the Institute of Automatic Control, University of Kaiserslautern, 67653 Kaiserslautern, Germany \{sschneider, litz\}@eit.uni-kl.de

${ }^{2}$ Jean-Jacques Lesage is with the Laboratoire Universitaire de Recherche en Production Automatisée (LURPA) - Ecole Normale Supérieur de Cachan, 61, Avenue du Président Wilson, 94235 Cachan Cedex, France lesage@lurpa.ens-cachan.fr
}

and the condition template approach. Another identification attempt for timed DESs is given in [7]. Based on a set of observed data a timed automaton is build. The transitions are constrained by time intervals. The identified interval bounds correspond to the minimum and maximum observation times. Timed Petri Nets for modeling DES are identified in [8]. The transition firing periods are assumed as normally and exponentially distributed. Confidence intervals are determined for each distribution with respect to a specified risk level.

This work focuses on identification of a timed automaton for model-based fault diagnosis in DES. The identified automaton represents the fault-free system behavior. Previous works using this model class did not investigate the identification of timed behavior in terms of real DES. The contribution of this paper is a new generic method for time guard determination of large-scale DESs affected by disturbances. Known approaches for time guard determination relay on minimal and maximal time observations or use expert knowledge of the timed DES behavior. In this work it is shown that their performance is limited under realistic conditions with large applications, unknown distributions and distorted data. The new method is presented requiring little a-priori knowledge about the timed behavior of the DES. For evaluation, particular attention is payed to false and missed alarms with fault detection.

The paper is structured as follows. In section II the DES framework is introduced. The timed automaton is defined in section III. Section IV presents the main contribution of this work. The timed identification approach is explained in detail with focus on time guard determination. The theoretical results are evaluated using a real industrial system in section $\mathrm{V}$. In section VI the results are concluded.

\section{Discrete Event Systems}

The DES framework is applied to describe the dynamic behavior of plant and logic controller. Fig. 1 illustrates

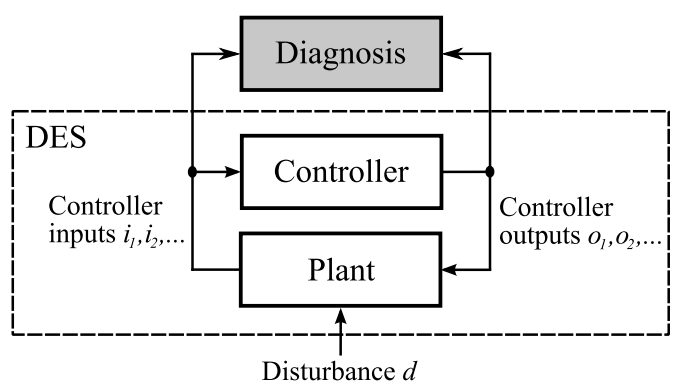

Fig. 1. Closed-loop DES with diagnosis 
the closed-loop DES with diagnosis. Plant and controller are connected via controller inputs $i_{1}, i_{2}, \ldots$ and outputs $o_{1}, o_{2} \ldots$. The input and the output vector are given respectively by $i=\left(i_{1}, i_{2}, \ldots\right)$ and $o=\left(o_{1}, o_{2}, \ldots\right)$. Controller inputs and outputs (I/Os) compose the $\mathrm{I} / \mathrm{O}$-vector $u=$ $(i, o)$ of the DES. For fault detection the $\mathrm{I} / \mathrm{O}$-vector $u$ is forwarded to the diagnosis unit. The controller is assumed to be deterministic, i.e. the controller computes for a specific sequence of inputs a specific sequence of outputs. The plant can be non-deterministic and affected by an immeasurable disturbance $d$.

The DES is considered as a black-box. By observing the I/O-vector $u$ the behavior of the DES is determined. Only state transitions of the DES leading to a change of $u$ are observable. Transitions which do not lead to a change within $u$ are unobservable and not further considered. They are not applicable since the presented approach is data based and relies on observations. The obtained data from the DES are sequential occurrences of discrete events at discrete points in time.

Formal languages describe the behavior of a DES based on event sequences. The finite set of symbols $E$ represents the alphabet of the formal language. The symbols are determined by the I/O-vector $u$ of the DES. Each I/O-vector $u_{i} \in E$ corresponds to a unique symbol. A finite sequence of symbols is given by $u=u_{1}, u_{2}, \ldots$ with $u_{j+1} \neq u_{j}$. This represents the structural information of the DES obtained with an event sequence. The corresponding finite time attribute sequence is given by $t=t_{1}, t_{2}, \ldots$ where $t_{j} \in \mathbb{R}_{0}^{+}$and $t_{j+1}>t_{j}$. This represents the time information obtained with an event sequence. The timed alphabet $E_{t} \subseteq E \times \mathbb{R}^{+}$contains pairs of symbols and time attributes $\left(u_{j}, t_{j}\right) \in E_{t}$. Each symbol is attributed with one absolute time value corresponding to the real time. A timed word $w$ is a finite sequence of timed symbols $w=\left(u_{1}, t_{1}\right),\left(u_{2}, t_{2}\right), \ldots$ with $\left(u_{j}, t_{j}\right) \in E_{t}$. Words $w$ of arbitrary length are obtained based on sequences of timed symbols. The timed language $L$ is a set of words $w$ over $E_{t}$ generated by the DES. An example of two different timed symbol sequences is given in Fig. 2. Multiple time attributes of one symbol are different $\left(e . g t_{1}^{A} \neq t_{5}^{A}\right)$ because of the continuous running time.

\section{TIMEd Model}

The model applied in this work is the Timed Autonomous Automaton with Output (TAAO). This timed automaton is especially suitable to represent the structural and timed behavior of a closed-loop DES. The TAAO is defined by the eight-tuple

$$
T A A O=\left(X, x_{0}, \Omega, \lambda, C, m, T T, T G\right),
$$

with the finite set of states $X$, the initial state $x_{0}$, the output alphabet $\Omega$, the output function $\lambda$, the finite set of clocks $C$, the clock mapping function $m$, the finite set of timed transitions $T T$ and the finite set of time guards $T G$.

The output function $\lambda: X \rightarrow \Omega$ maps to each state of $X$ an element of the output alphabet $\Omega$. The clock mapping function $m: X \rightarrow C$ maps to a state $x_{i} \in X$ one clock $c_{i} \in$

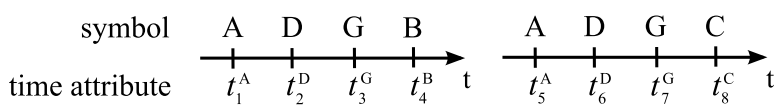

Fig. 2. Example of two DES sequences

$C$ with $1 \leq i \leq|X|$, where $|X|$ denotes the number of states. The mapping is bijective. The set of timed transitions is given as $T T \subseteq X \times T G \times C \times X$. Set $T T$ contains combinations of a source state $x \in X$, a time guard $\operatorname{tg}\left(x, x^{\prime}\right) \in T G$, a clock reset $v\left(x^{\prime}\right):=0$ and a target state $x^{\prime} \in X$. The transition between $x$ and $x^{\prime}$ is labeled with $\left(x, \operatorname{tg}\left(x, x^{\prime}\right), v\left(x^{\prime}\right), x^{\prime}\right)$. The clock valuation function $v: X \rightarrow \mathbb{R}_{0}^{+}$maps to the clock $c_{i} \in C$ of a state $x_{i} \in X$ a real value. The value of clock $c_{i}$ indicates the elapsed time after activating $x_{i}$. The set of time guards $T G$ contains Boolean conditions $\operatorname{tg}\left(x, x^{\prime}\right)=$ $\left(v(x) \geq \tau_{x, x^{\prime}}^{l o}\right) \wedge\left(v(x) \leq \tau_{x, x^{\prime}}^{u p}\right)$ where $x$ is a source and $x^{\prime}$ the corresponding target state of a transition. A time guard returns true if $v(x) \in\left[\tau_{x, x^{\prime}}^{l o}, \tau_{x, x^{\prime}}^{u p}\right]$ and otherwise false. The lower time guard limit is denoted as $\tau_{x, x^{\prime}}^{l o}$ and the upper time guard limit as $\tau_{x, x^{\prime}}^{u p}$ with $\tau_{x, x^{\prime}}^{l o}, \tau_{x, x^{\prime}}^{u p} \in \mathbb{R}_{0}^{+}$.

The TAAO is defined in accordance with the timed automaton given in [9] and the Non-Deterministic Autonomous Automaton with Outputs (NDAAO) given in [5]. Identification and fault detection is performed without any active interventions in the operating system. This motivates an automaton without an input alphabet but with outputs. The TAAO represents a timed autonomous event generator that is able to simulate the external behavior of a DES. Hence, it constitutes an appropriate model for fault detection purpose.

\section{Time Guard Determination}

\section{A. Identification Problem}

Model-based fault detection requires a model that is able to represent the structural and time behavior of the closedloop DES. Since the number of inputs $i$ and outputs $o$ of the considered system class is very high $(>100)$ manual modeling is very time-consuming and therefore impractical. Furthermore, only very little knowledge about controller and plant of the considered system class may be available. To overcome these problems an identification approach is chosen to determine a model of the DES based on observed system evolutions.

The aim of identification is to determine a TAAO that reproduces the original timed language $L_{\text {orig }}$ of the DES. Language $L_{\text {orig }}$ represents the fault-free behavior of the DES. The term fault-free describes the accepted system behavior. Disturbances may lead to small changes in the behavior which are not considered as faulty. Including this behavior into the model helps to avoid a large number of false alarms.

In Fig. 3 the relations between the languages are illustrated. Identification is performed based on the observed language $L_{o b s}$. Since the observation horizon is always finite the observed language may be incomplete $L_{o b s} \subseteq L_{\text {orig }}$. Identification yields the language $L_{\text {ident }}$. In general, the resulting identified language is not equal to the original 


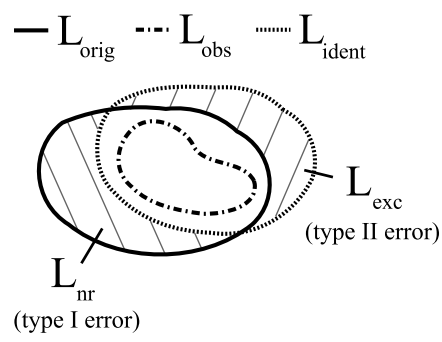

Fig. 3. Original and identified languages

one $L_{\text {ident }} \approx L_{\text {orig. }}$. The consequence is a non-reproducible language $L_{n r}=L_{\text {orig }} \backslash L_{\text {ident }}$ and an exceeding language $L_{\text {exc }}=L_{\text {ident }} \backslash L_{\text {orig. }}$. The non-reproducible language $L_{n r} \subset L_{\text {orig }}$ is a part of the original language that can not be reproduced by the identified model. This set denoted as type I error leading to false alarms with fault detection. The exceeding language $L_{\text {exc }} \subset L_{\text {ident }}$ is not part of the original language $L_{\text {orig }}$ but of the identified language $L_{i d e n t}$. This set leads to a number of undetectable faults and is denoted as type II error.

To improve fault detection the number of false alarms and undetectable faults has to be reduced. Therefore, appropriate identification strategies are required. In the following the focus is on determination of time guards in identified models with respect to the errors type I and type II.

\section{B. Identification}

Model identification is performed in two successive steps. First, the structure of the automaton and second, the time related components are identified. The data base for identification contains a set of system evolutions representing $L_{\text {obs }}$. An evolution is given as $\sigma_{h}=$ $\left(\left(u_{h}(1), t_{h}(1)\right),\left(u_{h}(2), t_{h}(2)\right), \ldots,\left(u_{h}\left(l_{h}\right), t_{h}\left(l_{h}\right)\right)\right)$ where $\left(u_{h}(j), t_{h}(j)\right) \in E_{t}$ is the $j$-th time attributed symbol in the $h$-th system evolution of length $l_{h}$. The set of all observations is denoted as $\Sigma=\left\{\sigma_{1}, \ldots, \sigma_{h}, \ldots, \sigma_{p}\right\}$ with $p$ observed evolutions. It is assumed that the initial character is the same for all system evolutions $\sigma_{i}(0)=\sigma_{j}(0)$ for all $i \neq j$.

Structural identification is performed based on the untimed language $L_{u}$. The untimed language $L_{u}$ results from the projection $\operatorname{proj}: L \rightarrow L_{u}$ with $\operatorname{proj}\left(\left(u_{1}, t_{1}\right),\left(u_{2}, t_{2}\right), \ldots\right)=$ $u_{1}, u_{2}, \ldots$ for all $w \in L$. The time attributes of each timed word $w$ are masked and only the symbols $u_{1}, u_{2}, \ldots$ are used. Consequently, $L_{u}$ represents a set of untimed words $w_{u}$ over $E$. The time guards $\operatorname{tg}\left(x, x^{\prime}\right)$ with the transitions $\left(x,\{\}, m\left(x^{\prime}\right), x^{\prime}\right) \in T T$ of the structurally identified TAAO are not determined so far thus the set of the transition label is empty. The applied algorithm for structural identification is given in [10].

Time identification includes time span allocation and time guard determination. Basically, with time span allocation the observed time attributes of all $\sigma_{h} \in \Sigma$ are assigned to the corresponding transitions of the model. The observed sequences of time attributes are given as $t_{h}=\left(t_{h}(1), t_{h}(2), \ldots, t_{h}\left(l_{h}\right)\right)$ for all $\sigma_{h} \in \Sigma$. A time span $\delta(j)$ between two successive symbols $(u(j), t(j))$ and $(u(j+1), t(j+1))$ is denoted as

$$
\delta(j)=t(j)-t(j-1) .
$$

It is the relative time in the $j$-th event step. The ordered sequence $\delta_{h}$ of all time spans for a given system evolution $\sigma_{h}$ is $\delta_{h}=\left(\delta_{h}(1), \delta_{h}(2), \ldots, \delta_{h}\left(l_{h}-1\right)\right)$ with length $l_{h}-1$. For each time span $\delta_{h}(j)$ the corresponding transition of the model has to be determined. Therefore, the state trajectory $\bar{x}=x_{0}, x_{1}, \ldots, x_{\left|\sigma_{h}\right|}$ over $X_{h} \subseteq X$ of the structurally identified TAAO is considered. The state trajectory $\bar{x}$ is unambiguous for $\sigma_{h}$ where $\lambda\left(x_{i}\right)=u_{h}(j), x_{i} \in X_{h}$ and $u_{h}(j) \in \sigma_{h}$. The proof is given in [10]. Within the state trajectory $\bar{x}$ the transition between $x_{i}$ and $x_{j}$ can be unambiguously determined if and only if $x_{i}, x_{j} \in X_{h}$. With given $x$ the active transition $t t_{x, x^{\prime}}^{a c t}$ within $\bar{x}$ is determined as

$$
\begin{aligned}
t t_{x, x^{\prime}}^{a c t}=\left(\left(x,\{\}, m\left(x^{\prime}\right), x^{\prime}\right)\right. & \in T T: \\
& \exists_{x^{\prime} \in X_{h}}\left(u(j)=\lambda\left(x^{\prime}\right)\right) .
\end{aligned}
$$

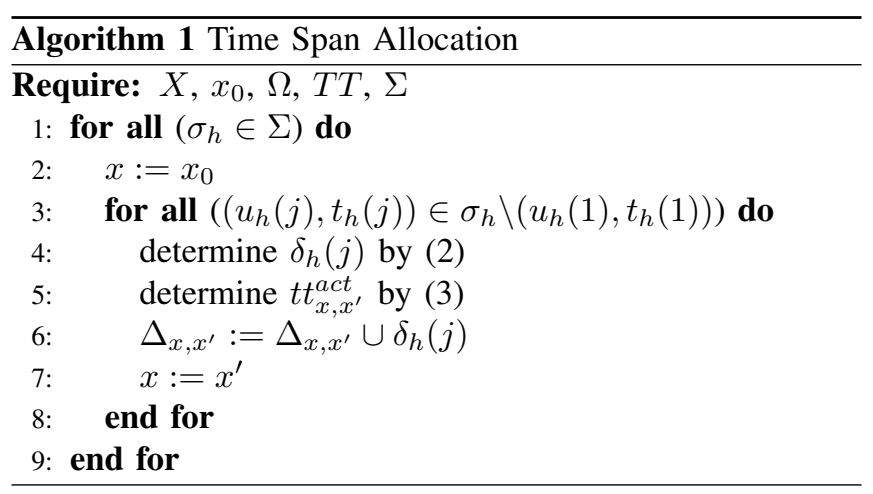

Algorithm 1 outlines the time span allocation procedure. It is performed for all observed sequences $\sigma_{h}$. In the first step state $x$ is initialized. For each observed timed symbol $\left(u_{h}(j), t_{h}(j)\right)$ of sequence $\sigma_{h}$ the time span $\delta_{h}(j)$ is calculated. The first symbol $\left(u_{h}(1), t_{h}(1)\right)$ is excluded. The active transition $t t_{x, x^{\prime}}^{a c t}$ is determined and the time span $\delta_{h}(j)$ is added to the set $\Delta_{x, x^{\prime}}$ of transition $t t_{x, x^{\prime}}^{a c t}$. State $x$ is set as the target state of the transition $x^{\prime}$ and the allocation procedure is performed again up to the end of sequence $\sigma_{h}$. The result of time allocation are the sets of observed time spans $\Delta_{x, x^{\prime}}$ for each transition.

The time span allocation procedure is demonstrated using the example of Fig. 2. The system evolutions are given as $\sigma_{1}=((A, 6),(D, 10),(G, 25),(B, 31))$ and $\sigma_{2}=$ $((A, 36),(D, 42),(G, 52),(C, 63))$. Structural identification is performed according to [10] and the sequences of time spans are determined as $\delta_{1}=(4,15,6)$ and $\delta_{2}=(6,10,11)$.

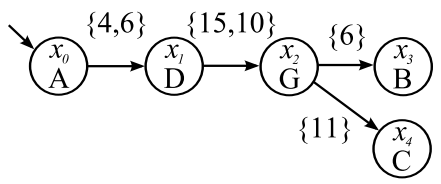

Fig. 4. Example TAAO with allocated time spans 
The resulting structural identified TAAO with allocated time spans is illustrated in Fig. 4. The states $x_{0}, \ldots, x_{4}$ are labeled with their outputs. At the transitions the observed time spans $\Delta_{x, x^{\prime}}$ after time span allocation are listed.

\section{Time Guard Determination Using Existing Attempts}

Time guard determination calculates the lower $\tau_{x, x^{\prime}}^{l o}$ and upper time bounds $\tau_{x, x^{\prime}}^{u p}$ of each transition based on the set of observed time spans $\Delta_{x, x^{\prime}}$. For reasons of clarity the explanation is focused on a single transition in the following. The two approaches commonly used are:

Min/Max: The time guard is determined by

$$
\begin{aligned}
\tau^{l o} & =\min (\Delta)), \\
\tau^{u p} & =\max (\Delta)) .
\end{aligned}
$$

There is no parameter to adjust the computation.

Normal Distribution: It is assumed that each observed time span $\Delta$ of the DES is normally distributed with mean $\mu$ and standard deviation $s$. The density function of the normal distribution $N(\mu, s)$ for $\Delta$ of the transition is given as

$$
\Phi(\delta, \mu, s)=\frac{1}{\sqrt{2 \pi} s} e^{-\frac{(\delta-\mu)^{2}}{2 s^{2}}} .
$$

Based on $\Delta$ the mean $\mu$ is estimated applying

$$
\hat{\mu}=\frac{1}{n} \sum_{i=1}^{n} \Delta(i)
$$

and the estimator of the standard deviation $s$ is

$$
\hat{s}^{2}=\frac{1}{n-1} \sum_{i=1}^{n}(\Delta(i)-\hat{\mu})^{2},
$$

where $n=|\Delta|$ holds for both estimations. The time guards are determined as

$$
\begin{aligned}
\tau^{l o} & =\hat{\mu}-\nu \cdot \hat{s}, \\
\tau^{u p} & =\hat{\mu}+\nu \cdot \hat{s}
\end{aligned}
$$

with parameter $\nu \in \mathbb{N}$ to adjust the size of the interval according to an expected number of observations within these bounds (e.g. $\nu=3$ yields $99.74 \%$ of all observations are expected within $[\hat{\mu}-3 \hat{s}, \hat{\mu}+3 \hat{s}])$.

\section{Time Guard Determination using Skewness Adaption}

Several problems may arise with the state of the art time guard determination approaches. Min/Max is based on the assumption that with a sufficient large observation horizon all timed behavior of the DES is observed. For large-scale systems the required observation horizon would be impractical large. Furthermore, with fault detection the observed times are affected by stochastic disturbances. Disturbed observations close to the identified time bounds may lead to false alarms. With the second approach the observed time spans of all transitions have to be normally distributed.

To handle disturbances and asymmetry of the observed time data the new attempt Skewness Adaption is introduced. Skewness Adaption does not rely on a specific distribution, provides a certain degree of uncertainty in the observed data, and takes the skewness of the time span distributions into account. The applied estimator for a robust skewness estimation after [11] is given as

$$
Q S=\frac{\delta_{0.875}-\delta_{0.5}-\left(\delta_{0.5}-\delta_{0.125}\right)}{\delta_{0.875}-\delta_{0.125}},
$$

where $\delta_{q}$ denotes the $q$-th quantile of the considered time span distribution. The estimator calculates a normalized skewness value $-1 \leq Q S \leq 1$. A distribution is considered as right-skewed if $Q S \geq \alpha$, left-skewed if $-Q S \geq \alpha$ and non-skewed if $|Q S|<\alpha$. The limit $\alpha$ is introduced to distinguish between non-skewed and clearly skewed distributions.

The time guard for a right-skewed distribution $(Q S \geq \alpha)$ is determined as

$$
\begin{aligned}
\tau^{l o} & =\min (\Delta))-\gamma \cdot \epsilon(\max (\Delta)-\min (\Delta)), \\
\tau^{u p} & =\max (\Delta))+\epsilon(\max (\Delta)-\min (\Delta)) ;
\end{aligned}
$$

for a left-skewed distribution $(-Q S \geq \alpha)$ as

$$
\begin{aligned}
\tau^{l o} & =\min (\Delta))-\epsilon(\max (\Delta)-\min (\Delta)), \\
\tau^{u p} & =\max (\Delta))+\gamma \cdot \epsilon(\max (\Delta)-\min (\Delta)) ;
\end{aligned}
$$

and for a non-skewed distribution $(|Q S|<\alpha)$ as

$$
\begin{aligned}
\tau^{l o} & =\min (\Delta))-\epsilon(\max (\Delta)-\min (\Delta)), \\
\tau^{u p} & =\max (\Delta))+\epsilon(\max (\Delta)-\min (\Delta)),
\end{aligned}
$$

where $\epsilon \in \mathbb{R}^{+}$denotes the extension and $\gamma \in \mathbb{R}^{+}$the adaption parameter. Skewness Adaption adds a tolerance $\epsilon(\max (\Delta)-\min (\Delta))$ to the minimum and maximum observed time span of a distribution. This increases the robustness of fault detection against the influence of disturbances. In the case of a skewed distribution the time bound at the side with the short tail is truncated using $\gamma$ (e.g. the lower bound $\tau^{l o}$ with a right-skewed distribution). This refers to the adaption ability of the method. It enables customization of the time guards with respect to the skewness property of the distributions. The time guard is finally determined as $\left[\max \left(0, \tau^{l o}\right), \tau^{u p}\right]$.

\section{E. Type I and II error}

Using identified models with fault detection leads to errors of type I and II. The aim is to detect faults precisely, i.e. with minimal number of false alarms and missed faults. The fault detection errors are defined as follows:

Type I error: False alarms $F_{I}$ : Rejecting an acceptable timed system behavior.

Type II error: Non-detectable faults $F_{I I}$ : Accepting a faulty timed system behavior.

Type I error $F_{I}$ represents the number of fault alarms which arise with fault detection using the identified model to simulate the accepted system behavior. It can be evaluated with an appropriate data base. The determination of the error type II is much more challenging. To quantify the time guard determination methods the probability of a type II error $P\left(F_{I I}\right)$ is considered. In Fig. 5 an example distribution of a transition is illustrated. It is assumed that an upper limit $\delta=2 \max (\Delta)-\mu$ exists where an observation during on-line fault detection beyond this time limit always corresponds to 


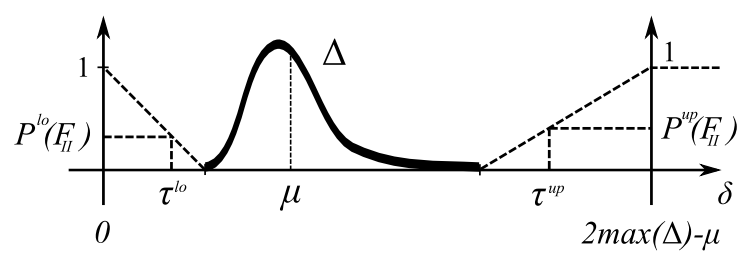

Fig. 5. Probability consideration of type II error

fault. The value depends on the considered application and is motivated by expert knowledge. Furthermore, it is assumed that occurrences of time spans are uniformly distributed over $0 \leq \delta \leq \min (\Delta)$ and $\max (\Delta) \leq \delta \leq 2 \max (\Delta)-\mu$. The conditional probability of an error type II for $\tau^{l o} \leq \delta^{\text {obs }} \leq$ $\min (\Delta)$ of one distribution is determined as

$$
\begin{aligned}
P^{l o}\left(F_{I I}\right) & =P\left(F_{I I} \mid \tau^{l o} \leq \delta^{o b s} \leq \min (\Delta)\right) \\
& =-\frac{1}{\min (\Delta)} \tau^{l o}+1
\end{aligned}
$$

and for $\max (\Delta) \leq \delta^{o b s} \leq \tau^{u p}$ as

$$
\begin{aligned}
P^{u p}\left(F_{I I}\right) & =P\left(F_{I I} \mid \max (\Delta) \leq \delta^{o b s} \leq \tau^{u p}\right) \\
& =-\frac{1}{\max (\Delta)-\mu}\left(\tau^{u p}-\max (\Delta)\right),
\end{aligned}
$$

where the observed time span $\delta^{o b s}$ leads to the detection of an abnormal behavior. The probability of a type II error $P\left(F_{I I}\right)$ related to a detection is calculated as

$$
P\left(F_{I I}\right)=\frac{1}{2}\left(P^{l o}\left(F_{I I}\right)+P^{u p}\left(F_{I I}\right)\right) .
$$

The probability $P\left(F_{I I}\right)$ is a relative measure to benchmark the different time guard determination methods against each other. The larger the gap between $\left(\tau^{l o}, \min (\Delta)\right)$ and $\left(\max (\Delta), \tau^{\max }\right)$ the higher the probability that an observed behavior within these bounds corresponds to a system fault.

\section{Evaluation}

\section{A. System Description}

For evaluation of the time guard determination methods an industrial non wovens manufacturing system is applied. It is controlled by a Programmable Logic Controller (PLC) using 114 I/Os. The I/Os are represented in binary format. The identified DES fault detection model consists of 8 automata with disjoint I/O sets. Applying the introduced methods to DES models consisting of multiple automata is straightforward. The data base contains 810 system evolutions used for learning and evaluation purpose. A system evolution is a fault-free production cycle that represents accepted system behavior.

\section{B. Observations}

In Fig. 6 the lengths of the time span sets $\left|\Delta_{x, x^{\prime}}\right|$ for all transitions of all automata are illustrated. The transitions are numbered consecutively. Based on the applied structural identification algorithm a modeled transition is observed at least one time. The range from transition 186 to 282 is highlighted in the figure.

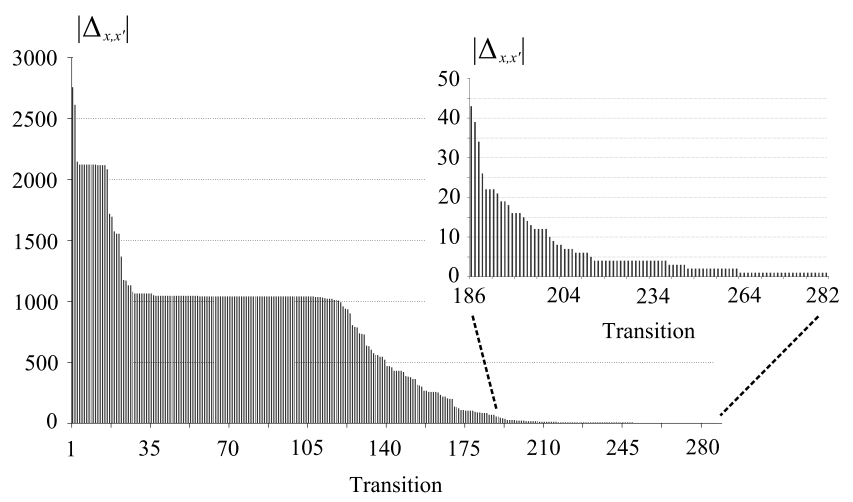

Fig. 6. Number of observations for each transition

To obtain reliable statistical results a minimum number of observations $n_{0}$ for each distribution is required. As larger $n_{0}$ is as more reliable the determined time guards. During validation $n_{0} \geq 50$ delivered reasonable results. Choosing $n_{0}=50$ implies that $66 \%$ of all transitions are considered for time guard determination. The time guards of all transitions where $\left|\Delta_{x, x^{\prime}}\right|<n_{0}$ are set to $\operatorname{tg}\left(x, x^{\prime}\right)=[0,+\infty]$.

\section{Time Guard Determination Results}

The time guard determination attempts from section IV are evaluated using the error type I and probability of type II error defined in subsection IV-E as criteria. For evaluation the transitions are separated into two groups with skewed and non-skewed distributions, respectively. Setting $\alpha=0.7$ leads to a separation where $12.8 \%$ of all considered time span distributions are considered as skewed on average. The value of $\alpha$ is empirically determined. A $k$-fold cross validation strategy is applied. The data base is partitioned into $k$ groups, where the $i$-th group is validation group $1 \leq i \leq k$ and all other groups $k-1$ are training groups. The result is the average of all $k$ validation runs. For all runs the global parameters are set to $n_{0}=50, \alpha=0.7$, and $k=3$.

For skewed distributions the average type I error is shown in Fig. 7 and the average probability of type II error in Fig. 8 for all $k$ validation runs. In Fig. 9 and 10 the errors obtained with non-skewed distributions are depicted. The smallest average number of false alarms is obtained with Skewness Adaption and the Normal Distribution approach.

The probability of type II error obtained with Skewness Adaption is smaller than for the Normal Distribution approach with $\nu=8$ while type I error is almost the same. Hence, the total performance resulting with Skewness Adaption is clearly better than with a Normal Distribution approach. According to definition $P\left(F_{I I}\right)=0$ using the Min/Max determination.

Focusing only the skewed distributions both Skewness Adaption attempts reveal the same number of false alarms while the probability of error type II is decreased by 0.02 with active adaption $\gamma=0.7$. This shows the benefit of adapting the time bounds with skewed distributions. In both parameterizations no adaption is calculated with non-skewed distributions. 


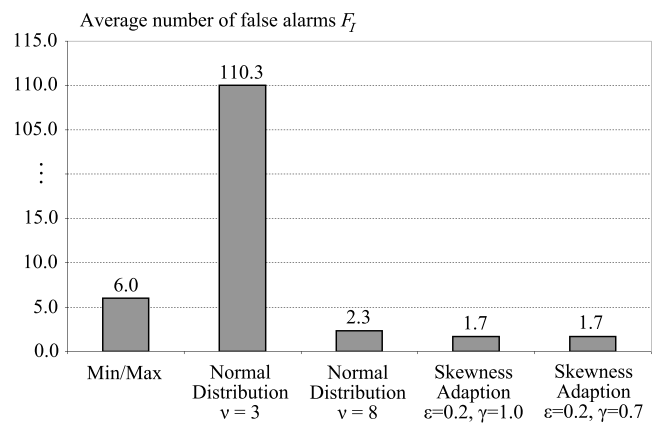

Fig. 7. Skewed distributions type I error

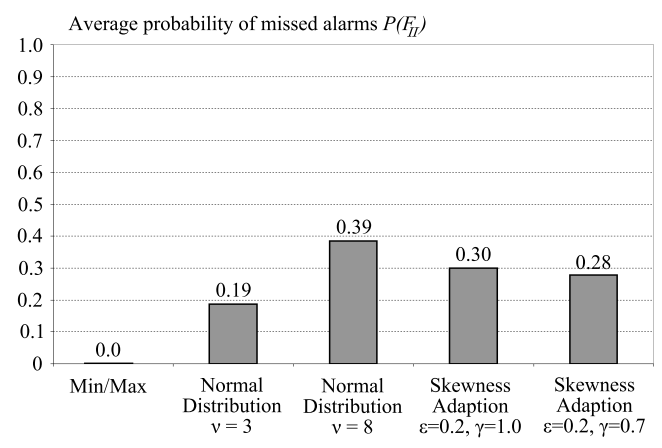

Fig. 8. Skewed distributions type II error

In summary, the Skewness Adaption attempt leads to an advantageous setting when a certain probability of type II error is acceptable and a small number of false alarms is desired. The selection of $\epsilon$ and $\gamma$ depends on the acceptable number of false alarms. They can be determined by evaluating the observed data.

\section{CONCLUSION}

This work presents a generic method for the determination of timed transitions in models of large-scale systems. The timed identification consists of time span allocation and time guard determination. For time guard determination with skewed and disturbed time data the new approach Skewness Adaption is introduced. It is evaluated and benchmarked with the state of the art approaches: Min/Max and assuming normally distributed data. The results show that with Skewness Adaption very low numbers of false alarms can be achieved at the expense of moderate probabilities of missed detections. The method works with skewed and non-skewed time span distributions likewise. Min/Max results in a low probability of missed detections with the disadvantage of high numbers of false alarms which are not acceptable for fault detection in industrial systems. The overall worst results are obtained with methods that assume normally distributed time spans.

For real applications requiring a small number of false alarms the Skewness Adaption method is the best choice. An advantageous trade-off between false alarms and missed faults is achieved. The resulting models are very appropriate for fault detection in DESs.

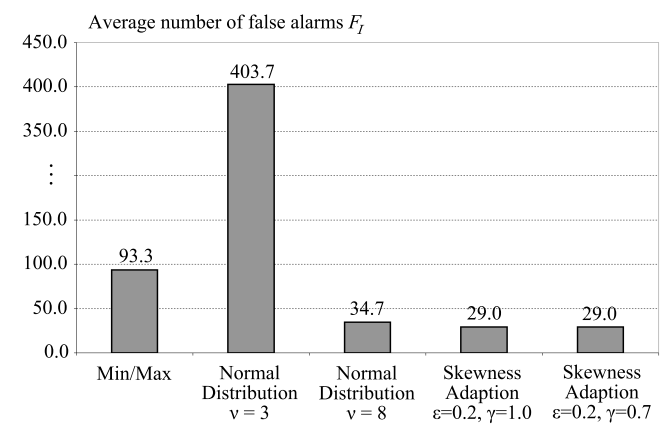

Fig. 9. Non-skewed distributions type I error

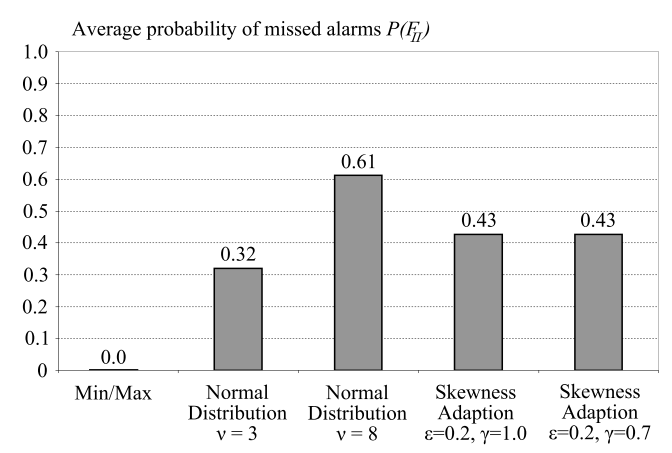

Fig. 10. Non-skewed distributions type II error

\section{REFERENCES}

[1] D. Pandalai and L. Holloway. Template Languages for Fault Monitoring of Timed Discrete Event Processes. IEEE Transactions on Automatic Control, vol. 45, no. 5, May 2000, pp. 868-882.

[2] S. R. Das and L. E. Holloway. Characterizing a confidence space for discrete event timings for fault monitoring using discrete sensing and actuation signals. IEEE Transactions on Systems, Man and Cybernetics - Part A: Systems and Humans, vol. 30, no. 1, January 2000, pp. 5266.

[3] M. Sampath, R. Sengupta, S. Lafortune, K. Sinnamohideen and D. Teneketzis. Failure Diagnosis Using Discrete-Event Models. IEEE Transactions on Control Systems Technology, vol. 4, no. 2, March 1996, pp. 105-124.

[4] S. Hashtrudi Zad, R. H. Kwong and W. M. Wonham. Fault Diagnosis in Discrete-Event Systems: Incorporating Timing Information. IEEE Transactions on Automatic Control, vol. 50, no. 7, July 2005, pp. 1010-1015.

[5] M. Roth, S. Schneider, J.-J. Lesage, and L. Litz. Fault detection and isolation in manufacturing systems with an identified discrete event model. International Journal of Systems Science, doi: $10.1080 / 00207721.2011 .649369$, in press.

[6] M. Danancher, J.-J. Lesage, and L. Litz. A comparison of three modelbased diagnosis approaches for industrial discrete event systems. 3rd International Workshop on Dependable Control of Discrete Systems (DCDS'11), June 2011, pp. 29-34.

[7] P. Supavatanakul, C. Falkenberg and J. Lunze. Identification of timed discrete-event models for diagnosis. Fourteenth International Workshop on Principles of Diagnosis, 2003, pp. 193-198.

[8] D. Lefebvre and E. Leclercq. Stochastic Petri Net Identification for the Fault Detection and Isolation of Discrete Event Systems. IEEE Transactions on Systems, Man and Cybernetics, vol. 41, no. 2, March 2011, pp. 213-225.

[9] R. Alur and D. L. Dill. A theory of timed automata. Theoretical Computer Science, vol. 126, 1994, pp. 183-235.

[10] M. Roth. Identification and Fault Diagnosis of Industrial ClosedLoop Discrete Event Systems. PhD thesis, University of Kaiserslautern, Logos Verlag Berlin, 2010.

[11] G. Brys, M. Hubert and A. Struyf. A comparison of some new measures of skewness. Developments in robust statistics, ICORS 2001, Springer, 2003, pp. 98-113. 